\title{
VANET: Framework, Challenges and Applications
}

\section{Kalaivani D}

\begin{abstract}
Vehicular Ad-Hoc Network (VANET) is one of the essential research field because of large increase in usage of vehicles on road. Many VANET applications are employed to improve road safety conditions, vehicle traffic, an emergency warning to vehicle drivers, collision avoidance and other non-safety applications for comfort. The main aim of these applications are to modernize the various processes associated with road traffic, vehicles, drivers, passengers and pedestrians by implementing smart transport systems. This study is to implement an intelligent vehicular transport design to improve the road safety, navigation and comfort. VANET communication technology is a combination of mobile vehicles with ad hoc networks. The purpose of this research is to predict and prevent road accidents by transmitting emergency messages using vehicular network technology. While transmitting emergency messages, the VANET has various challenges. They are High mobility of the vehicles, Dynamic topology, Wireless communication, Minimum transmission delay, Connectivity of the network, optimal usage of transmission power. These challenges leave the vehicular networking disconnected and make an exchange of information very difficult. Even though many researchers have done significant work during the last decade on vehicular networking, some problems have not yet been solved.

Keywords: Clustering, Intelligent Transport System, Medium Access Control, Routing Protocols, Vehicular Ad-hoc Networks, Wireless Access in Vehicular Environment.
\end{abstract}

\section{INTRODUCTION}

In today's world, the population is increasing and India is one of the most densely populated countries. The increase in population, globalization, relocation and innovation leads to congested road traffic conditions. Every day the road traffic is being highly congested due to greater population and increase in business ventures. The transport infrastructure is not enough for the country's progress, civilization and economy. The congested roads leads to all types of pollution, many social problems, and the increase in fuel price which causes road rage and fuel shortage pressure. Initially, the focus was to improve the transport infrastructure by constructing efficient highway roads. Lately, the focus has been shifted to in corporating electronic technology in mechanical and automotive engineering. The advancement in electronics

Manuscript received on 17 March 2021 | Revised Manuscript received on 27 March 2021 | Manuscript Accepted on 15 April 2021 | Manuscript published on 30 April 2021.

* Correspondence Author

Dr. Kalaivani D*, Department of Information Science and Engineering, New Horizon college of Engineering, Bengaluru, India. dkalaivanisai@gmail.com

(C) The Authors. Published by Lattice Science Publication (LSP). This is an open access article under the CC-BY-NC-ND license (http://creativecommons.org/licenses/by-nc-nd/4.0/) technology, leads to construct vehicles by embedding them with sensors and many other smart devices such as global positioning system tracker, driving assistant and security camera for road safety and comfort. Intelligent Transportation System (ITS) is a concept, which combines various technologies namely computer, electronics and communication to enhance the road transport system. ITS is extensively vital for hassle-free and secure road transportation in the current world.

The cutting-edge technology in the ITS field enables to develop the safety, comfort and the sustainability of the networks. The potentials are infinite as the human involvement can be completely automated by fine-tuning the real time monitoring and the performance of the network on road can be. The historical data can be taken for analysis through data analytics real-time tools. Then road administrator constructs an innovative strategy to solve the real problems in the smart transport system. The wireless service protocols provide resourceful and pioneering solutions to acquire the objectives of smart transport systems. It is a system to resolve the road traffic issues to avoid congestion, accidents and environmental issues.

\section{INTELLIGENT TRANSPORTATION SYSTEM}

ITS is a combined system that implement sensors, information and communication technologies to enhance the road traffic standards, safety and comfort of the vehicles, to minimize the time and energy. The location information is implemented into vehicles to manage the traffic. ITS is a state-of-the-art technology to improve the transportation system, by using sensors, digital mapping, video monitoring and by sending messages.

The data obtained is processed, analyzed, evaluated and finally the information is distributed to the road users. ITS is a combined application to optimize the road comfort, mobility and road safety. These applications analyse, control, sense and communicate to manage the traffic, maximize the benefits of public, private and commercial transportation system and minimize the environmental influence. This smart transport system is needed to reduce the road accident rates, to deal with increasing congestion, to minimize the travel duration, to address poor road development, and many more. The various technologies involved in intelligent transport system are data retrieval, data processing, data communication and data distribution. For the data retrieval process, traffic is monitored with the help of traffic sensors such as radar and closed circuit television. The retrieved information is processed, verified and combined in the data management center. Then, data is communicated through wire line, wireless or fibre optics technologies.

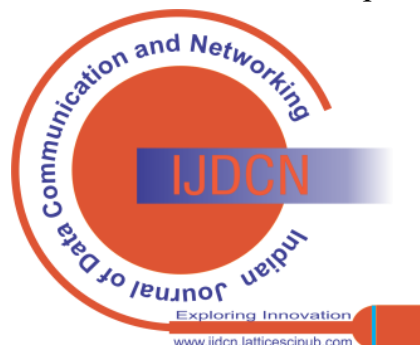


Finally, the data is distributed to ease the vehicle traffic, safety and environment.

ITS is a state-of-the-art technology to improve the transportation system, by using sensors, digital mapping, video monitoring and by sending messages.

\section{A. Framework of ITS}

In the ITS framework, many layered fields are maintained to support the primary technologies such as locating, sensing and information and communication technology. There are many applications for making use of this technology such as safe driving, road traffic control and pedestrian services. Apart from these technologies, the framework consists of control and map technologies. All these technologies support road safety, efficiency in all ways, environment and energy system and convenience and recreation features.

All these are platform-oriented applications with system implementation consisting of $\mathrm{N}$ number of systems with $\mathrm{N}$ number of applications associated with the common platform. Fig. 1 shows the contents of the intelligent transport system.

ITS consists of the intelligent infrastructure and the intelligent vehicles. The infrastructure is composed of the components such as metropolitan, rural and commercial vehicles. The recent advancements include the cloud infrastructure, which can send there all time information to the intelligent vehicles. Automated vehicles are categorized as automobiles, public transport vehicles, trucks and emergency or other special vehicles. The dissemination of emergency messages are broadcasted according to the intelligent vehicles category.

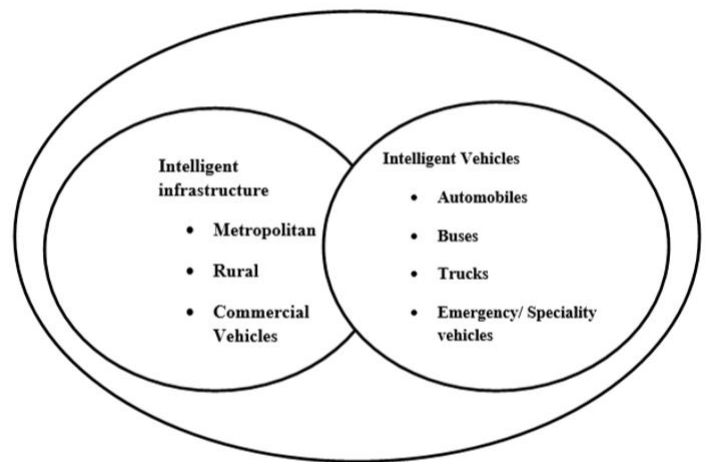

Fig 1: Intelligent Transportation Systems

\section{B. Components of ITS}

The Traffic Management Centre (TMC) is the transport administration hub where data is collected, analyzed and combined to control and manage the complex transportation network. The various components in the TMC as discussed in Bawangaonwala et al. (2018) [1]. are listed below:

Data Acquisition: The system combines hardware and software to collect consistent data for the ITS actions. It is significant for real-time monitoring and planning. The various hardware equipment are sensors, GPS, cameras, automatic vehicle identifiers, automatic vehicle locators and servers to store enormous amount of data.

Data Transmission: Communication tools are used to transmit the traffic related information. Reliable communication between data collection unit to TMC and transmit travel related information to vehicle's on On Board Units.
Data Analysis: The process of data cleaning, data fusion and analysis. The data from the sensors and other collection devices must be verified. Inconsistent data must be removed and then, the cleaned and fused data is analyzed to predict the traffic information.

Traveler Information: Travel Advisory System is used for transmitting variable messages such as message signs, highway radio, internet, short messaging services, public radio announcement, television broadcast and other tools. These systems can provide real-time information such as travel time, travel speed, delays, accidents route closures, detours and work zone conditions etc.

\section{VANET}

\section{A. An Overview of VANET Technologies}

The recent enhancement in the wireless communication technologies such as IEEE 802.11p creates research interest in VANET field. In the VANET communication technology, a group of vehicles maintain a communication network without any controller for a particular requirement. The communication will be between the moving vehicles in an environment. The communication happens when a vehicle communicates with another vehicle directly or when a vehicle communicates with an infrastructure such as roadside units. VANETs are customized form of Mobile Ad-Hoc Networks (MANETs) that offers communication between vehicles and roadside units with an objective to provide safety and comfort transportation services.

VANETs are a state-of-the-art technology that offers many applications such as traffic management and road traffic congestion monitoring. To avoid traffic congestion due to road accidents, natural calamities etc, the vehicles on that particular route can take alternate routes by transmitting emergency messages to the nearby vehicles with the help of VANET communication technology. Apart from safety applications in VANETs, there are many applications for services such as payment, infotainment etc (Hartenstein and Laberteaux, 2010) [2]. These applications communicate with the infrastructure, people and other vehicles.

\section{B. VANET Architecture}

The VANET architecture consists of many components such as intelligent vehicles fixed with receivers, transceivers, on board units, roadside units, workstations, servers etc. The mobile unit domain, infrastructure domain and management domain are the various modules in VANET architecture. In the mobile unit domain, vehicles are fixed with smart devices for communication between vehicle to vehicle and vehicle to infrastructure. In the infrastructure domain, the roadside units and other transceivers maintain the vehicle communication. Vehicle to infrastructure model of communication is performed in this domain. In the management domain, the servers and surveillance applications perform tasks such as sending alter messages to the other vehicles in the communication range. Fig. 2 shows the basic architecture of VANET.

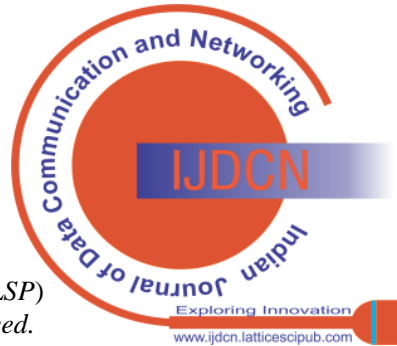


The data dissemination in VANET depends on vehicle to vehicle communication; vehicle to infrastructure communication and hybrid communication with a combination of V2V and V2I

(Ayyappan and Kumar, 2016) [3].

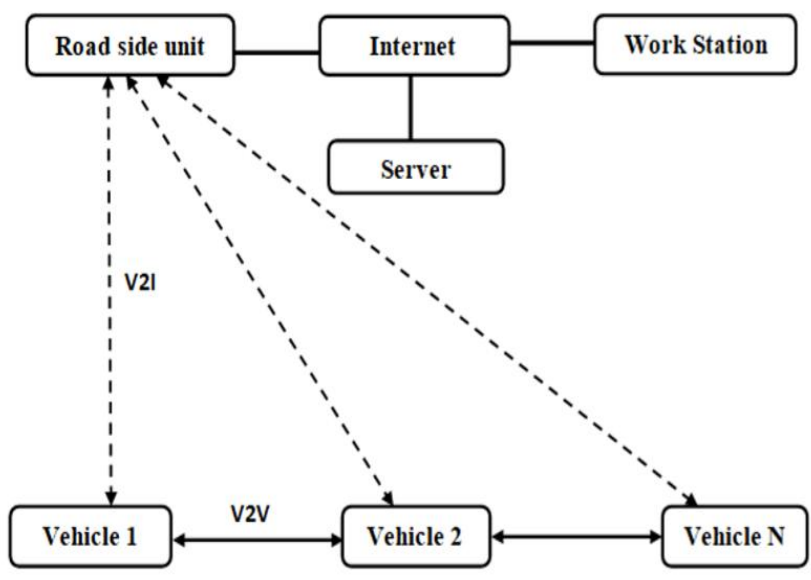

Fig 2. Basic Architecture of VANET

\section{Characteristics and Limitations of VANET}

Many characteristics and unique properties differentiate VANETs, which is a sub-category of MANETs. The unique properties are listed below as per explained in Ranjan and Ahirwar (2011) [4]. are as follows:

Extremely dynamic topology: The vehicles on highway road are moving at high speed making the topology dynamic due to multiple paths. The average speed Maintained by the vehicles will be out of order when the radio frequency between the vehicles is out of range. An efficient algorithm is proposed to maintain the link stability of highly mobile nodes.

Frequently disconnected network: The high speed of vehicles leads to dynamic topology and the lack of roadside units results in regular disconnection in the network. The link between the vehicles vanish due to dynamic topology and varying node density. Efficient routing protocols are required to adapt to frequent changes in topology and connectivity.

Patterned mobility modelling: A unique kind of mobility model is developed for traffic lights, speed limits, traffic scenarios and drivers. A mobility pattern is simulated and tested for VANET routing protocols. The movement and position of the vehicle are predicted based on the available roadmap models. The speed is again a huge challenge in developing the model for network design.

Propagation model: In this model, the static objects such as buildings, trees and other inferences of wireless communication, vehicles and multiple access points are considered.

Hard delay constraints: Delivering messages at the time of emergency without any delay is a crucial problem. The high data rate is also considered.

Unlimited battery power and storage: The nodes in the VANETs have unlimited battery power and storage. Energy consumption should be optimized for the efficient use of VANETs sensor networks.
On board sensors interaction: The mode of communication is performed through sensors. The nodes are equipped with sensors to provide the routing information.

\section{VANET Standards}

The common wireless communication standard in local area networks is IEEE 802.11. For the Dedicated Short Range Communication (DSRC) $75 \mathrm{MHz}$ frequency is allocated as shown in Fig .3 . Many public and private applications such as road traffic management, safety applications and vehicle entertainment applications use the DSRC standards. IEEE 802.11p is a standard for Wireless Access in Vehicular Environment (WAVE), which works on enhanced distributed channel access standards. This standard can be applied to VANETs for on board units and roadside units. IEEE 802.11p standard is used in the physical layer and Medium Access Control (MAC) layer. The interface functions in MAC are defined by the 802.11 standard. The MAC operations, vehicle to vehicle communications and vehicle to infrastructure communications are performed by this standard. The carrier sense multiple access with collision avoidance and orthogonal frequency division multiplexing techniques are included in IEEE 802.11p standard. To support high speed moving vehicles and to ease the communication method, WAVE standards were implemented. Real time traffic information and enhanced safety and road traffic are the advancements of the WAVE standard with the help of on board units and roadside units.

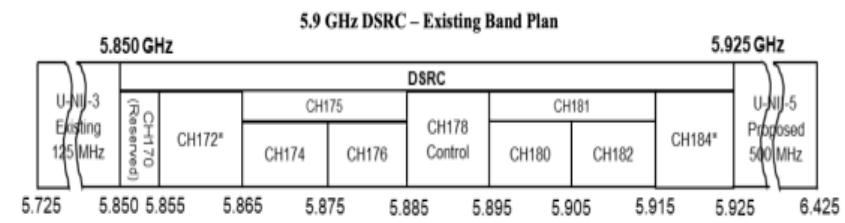

*Channels 172 and 184 are designated for public safety applications involving safety of life and property.

\section{Fig. 3. Dedicated Short Range Communication}

\section{E. Components of WAVE}

The WAVE architecture consists of IEEE 1609 standards with four sub-standards to perform the various issues and services. IEEE 802.11 operates in MAC and physical layers. The 1609 operates in the upper layers. Fig. 4 shows the various sub layers of IEEE 1609 is IEEE 1609.1, IEEE 1609.2, IEEE 1609.3 and IEEE 1609.4. IEEE 1609.1 works in the application layer to perform tasks such as interfacing, message formatting and services related to safety and non-safety applications. 1609.2 is implemented for network security. The various functions performed by these components are defining safety formats for messages, processing and exchanging messages and encrypting protocols for secure communication. 1609.3 works in the network and transport layer and takes care of the routing services and optimized protocol for data exchange in VANETs. 1609.4 is used for coordination to multiple channels in the DSRC. Operations such as switching between channels and tuning the same channel simultaneously can be performed [5]

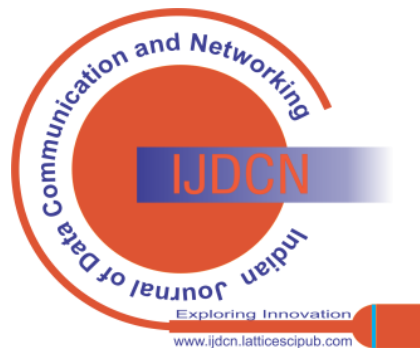




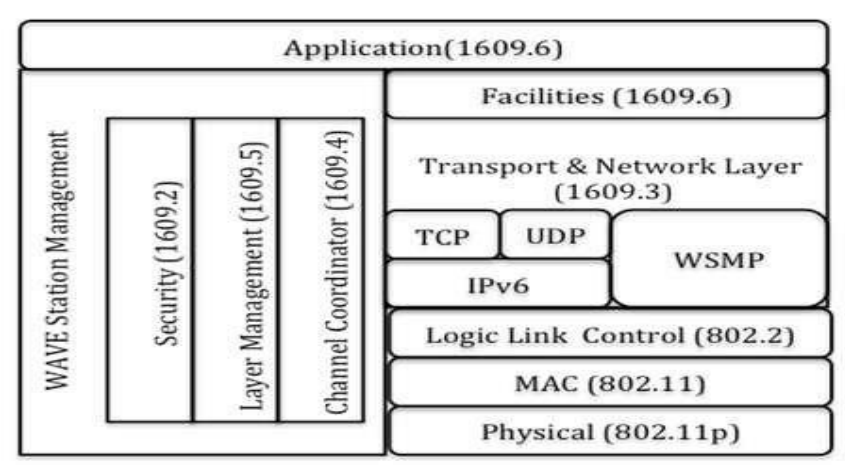

Fig. 4. Wave Architecture

\section{F. Challenges in VANET}

The various challenges in VANET are explained in [6] and categorized into vehicular security challenge, vehicular networks challenge and vehicular technical challenge.

- Vehicular Security Challenge- The VANET security challenge is considered based on the VANET architecture and the security protocols. Many security attacks and threats are faced by VANETs. The network attack is given high priority as the entire network is unsafe.

- Vehicular Networks Challenge- The vehicular networks challenge and opines as mobility the key idea of Ad Hoc networks as been explain in detail in [7] and [8]. Each node in the network is highly mobile and the vehicles in the network create connection and after few moments the connection is disconnected and this is one of the key challenges. The connectivity with the nodes is highly volatile. The authentication of every vehicle in the network will prevent attacks. Network scalability is another challenge due to the growing network.

- Vehicular Technical Challenge- The technical challenges are the technical obstacles taken care before deploying the VANET. Network management is the primary technical challenge due to high mobility, changing topology and changing channel condition. Uncontrolled network size creates congestion and collision. VANET communication use select magnetic waves and MAC design uses shared medium, which creates a huge impact to the environment. The life critical messages in the road safety application are been protected and secured.

\section{CLUSTERING TECHNIQUES IN VANET}

Clustering in VANETs is separating the network into diverse collection of vehicles. A cluster is an individual collection of vehicles in VANET technology. Each cluster consists of cluster head and cluster members. The communication takes place between the cluster members and different clusters. The clustering is needed to reduce the routing overheads, to improve the message delivery and to use the network bandwidth. The classified types of clustering into static and dynamic is explained in [9].

In the static cluster type, the clusters shift in similar direction and similar speed. There is no scope of scalability and does not require reconfiguring. The cluster design and forming is easy.
In the dynamic cluster type, clusters are formed dynamically with less time. The cluster heads are changed, highly mobile, reconfiguring happens based on the density and these types of clusters are scalable.

In [10] the exchange of information is performed by creating a backbone in the Backbone based clustering technique has been explained. The cluster head selection and message broadcast is dependent on the backbone. The k-hop is a backbone based clustering where the cluster structure is decided by the distance between the hops. The number of hops controls the distance between the cluster head and the cluster members. The multi-hop clustering algorithm forms the stable cluster. The dissemination of messages at standard interval occur to vehicles at the $\mathrm{N}$-hop distance. To enhance the scalability of the network protocols, vehicle clustering is performed in VANETs. The characteristics such as dynamic topology and the broken connections influence the cluster performance.

A challenging problem in vehicle cluster is creating and maintaining the stable cluster. The recent research has proved that the mobility based clusters enhance the stability of the cluster. The identification number metrics is applied instead of radio strength. To measure the performance of the protocol, parameters such as number of clusters, cluster head duration, cluster member duration, cluster head change rate and number of state change are measured.

The various cluster based routing protocols explained by [11] are given below :

\section{- Mobility based protocols}

The creation and selection of cluster head is determined by the mobility based on the lane and the speed. The various parameters such as vehicle position, direction and speed are used for selecting the clusters. The subcategories in the mobility based clustering protocols are direction based scheme and non-direction based scheme. In the direction based scheme, classification depends on the direction of vehicles on the road.

In the lane based clustering, the direction of the vehicle is considered as one of the parameter and re-clustering overhead is minimized because of the network topology.In the non-direction based scheme, the cluster and the cluster head is selected without the direction metrics. It considers selecting the least mobility node based on the neighboring nodes and varying speed in the network.

- Non mobility based protocols

The protocol depends on the density of the network and does not depend on the mobility aspect. The clusters are formed repeatedly and the gateway assists in the communication. The independent clustering technique dynamically selects the network gateway nodes based on the network topology. The cluster head in a particular cluster selects the network gateway nodes by specifying the time required. The network address of the neighboring network is compared with the own network address to avoid alter of the network gateway nodes.

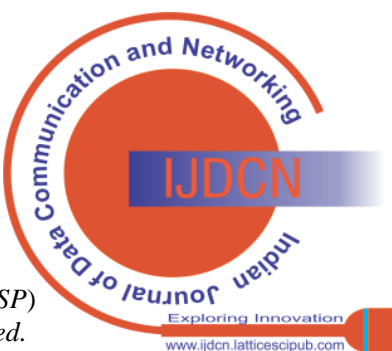




\section{- Certificate based protocols}

These protocols are used to protect the privacy. These protocols use the dynamic key infrastructure and are chosen for dynamic certificate authorities. The role of the certificate authority is to form the cluster heads and locate the hop distance from the cluster head to the other cluster members. The registration authority is responsible for certain nodes. In VANET infrastructure, each vehicle will receive numerous number of messages based on the certificate validation due to the time sensitivity. The certificate renovation scheme helps to improve the safety of the system. The roadside units replace the valid certificate with an invalid certificate. These certificates avoid unwanted information from other vehicles to improve the security.

- Vehicle based Clustering protocols

These protocols use the vehicle direction to select the cluster and the cluster head. The vehicle position and the direction is used for cluster formation. More than one cluster heads can be formed in this type of protocol. The only constraint is that the cluster head should be in opposite direction. This helps to enhance the conventional routing by location information for packet routing in the network. This technique minimizes the packet loss. The traffic is minimized if the nodes are selected. To minimize the global overhead and to maximize the clusters' lifetime, mobility pattern is used. The protocol enhances the lifetime of the cluster, minimizes the cluster head variations, minimizes the cluster re-affiliation and evades group re-clustering. Communication delay is increased, congestion and reliability issues and minimum data delivery happens when the message is broadcasted in groups.

- Efficiency and reliability based clusters

Intelligent transportation system focuses on the routing protocols, scalability and there liability in the VANETs. The applications of intelligent transport system serve characteristics such as irregular spatial distribution of vehicles, which affects the presentation of the clusters. There is a separate clustering algorithm to improve the link stability. The three clustering phases are selecting the clusterhead, creating the cluster and maintaining the cluster. Individual nodes are proposed to validate the network condition.

The various stages of clustering is explained in [12]. They are as follows :

\section{- Cluster formation}

The cluster is formed by creating a neighborhood relationship related with the broad casting region of the node. The inter cluster communication task and intra cluster communication task is performed based on the cluster radius. This can happen through one service channel and one channel is assigned as the control channel. The cluster formation happens when the slowest vehicle initiates the cluster formation by sending are quest signal. The stable neighboring vehicles participate in this procedure.

- Location and direction discovery

When the data packet is transmitted from the source to the destination and is not added in the routing table, then the location request is sent. The cluster head checks the location request and if it's the same then it rejects the packets. Otherwise, the location reply packet is sent to the source. When the destination node is not associated with the cluster head, then the address and the direction of the location request providing local information. The neighboring nodes use the

packet is cluster head. If it's in the same direction then its rebroadcasted to the location request packet and the neighboring cluster heads. Once data packet is transmitted back to the source node, the routing path is not maintained from the source to the destination. The path is defined in the location information provided by the source node. The path of the location request is different from the path of the location reply.

- Routing of data packets

Routing of data packets is associated with the location of the source and the destination, the direction and the neighbors of source and destination. Based on the locations, the packets are routed from the source to the destination. Based on the knowledge of the relative position packet, transmission occurs. Routing strategies such as the shortest path is calculated. Transmission is taking place based on the timing; if the time expires, then re-transmission of the data packets is initiated. This occurs due to packet loss or network disconnection.

- Maintenance of location information

The routing algorithm maintains and updates the location information before seeding the data packets. Once the packet reaches its destination, the acknowledgement packet is sent to the source node. The neighbor hoods are redefined according to the channels used for communication.

Once the cluster is formed, an appropriate cluster head is selected using the velocity, node degree, location and direction as the parameters. Mobility information for the nodes with stable neighbor is measured.

The vehicles continue to join the cluster, leave the cluster and two clusters merge to form single clusters due to the dynamic environment in VANET. The vehicles which come under different speed join the cluster at different timings. Based on the speed difference the cluster head acknowledges the cluster members.

A nodes Leaves the cluster only when a vehicle moves away from the specified radius and the connection gets disconnected. This information is maintained by the cluster-head. The merging of two clusters happens, when the cluster heads comes with in the broad casting range and the speed is within the threshold, which is predefined.

\section{SUMMARY}

In smart transport system for vehicles on highway roads, predicting and preventing road accidents by transmitting emergency messages to alert vehicles using wireless transmission technology is a significant problem.

One of the vital and complex tasks is to transmit the emergency.

\section{REFERENCES}

1. Bawangaonwala, M., Wadhwa, D., Nandeshwar, U. V., Dhurate, S., Ramteke, S. P., Bante, P. N. and Ansari, S. (2018), 'A review on development of intelligent transport system to compare with nagpur transport system', International Journal of Computer Science and Mobile Computing 7(4), 12-21. J. Clerk Maxwell, A Treatise on Electricity and Magnetism, 3rd ed., vol. 2. Oxford: Clarendon, 1892, pp.68-73.

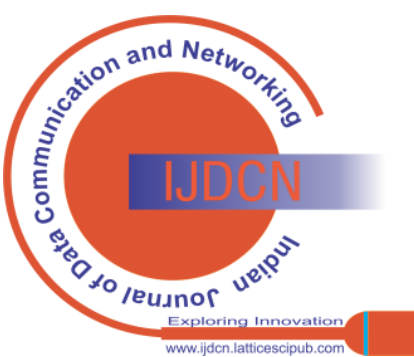


2. Hartenstein, H. and Laberteaux, K. (2010), VANET: vehicular applications and internetworking technologies, Wiley, UK. [CrossRef]

3. Ayyappan, B. and Kumar, P. M. (2016), Vehicular ad hoc networks (vanet): Architectures, methodologies and design issues, in 'Second International Conference on Science Technology Engineering and Management', pp. 177-180. [CrossRef]

4. Ranjan, P. and Ahirwar, K. K. (2011), Comparative study of vanet and manet routing protocols, in 'International Conference on Advanced Computing and Communication Technologies', pp. 517-523.

5. Eichler, S. (2007), Performance evaluation of the ieee $802.11 \mathrm{p}$ wave communication standard, in '66th Vehicular Technology Conference', pp. 2199-2203. [CrossRef]

6. Dinesh, D. and Deshmukh, M. (2014), 'Challenges in vehicle ad hoc network (vanet)', International Journal of Engineering Technology, Management and Applied Sciences 2(7), 76-88.

7. Samara, G., Al-Salihy, W. A. and Sures, R. (2010), Security issues and challenges of vehicular ad hoc networks (vanet), in "4th International Conference on New Trends in Information Science and Service Science', pp. 393-398. [CrossRef]

8. Rehman, S., Khan, M. A., Zia, T. A. and Zheng, L. (2013), 'Vehicular ad-hoc networks (vanets)-an overview and challenges', Journal of Wireless Networking and Communications 3(3), 29-38.

9. Kaur,P.andBhagat,N.(2016), 'A review on clustering in vanet', International Journal of Innovative Research in Computer and Communication Engineering 4(5), 9697-9700.

10. Zhang,Z., Boukerche, A. and Pazzi,R. (2011), A novel multi-hop clustering scheme for vehicular ad-hoc networks, in 'Proceedings of the 9th ACM international symposium on Mobility management and wireless access', pp. 19-26. [CrossRef]

11. Priyanka,T.andSharma,T.(2014),'Asurveyonclusteringtechniquesuse dinvehicular ad hoc network', International Journal of Advanced Computational Engineering and Networking 2(8), 4-9.

12. Rawashdeh,Z.Y. and Mahmud,S.M. (2012), 'A novel algorithm to form stable clusters in vehicular ad hoc networks on highways', EURASIP Journal on Wireless Communications and Networking 15(1), 1-13. [CrossRef]

\section{AUTHORS PROFILE}

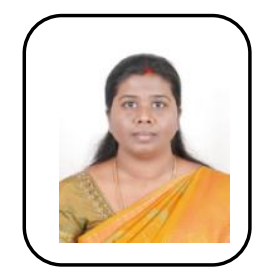

Dr. Kalaivani D, is currently working as an Associate Professor in the Department of Information Science and Engineering in New Horizon College of Engineering, Autonomous College Permanently affiliated to VTU. She earned her PhD in 2020 in the area of VANET from Vellore Institute of Technology under the supervision of Dr. Rajkumar S. She completed her Mtech in CSE from RVCE, VTU in 2008. She completed her B.E in CSE from VTU in 2005. Many paper publications have been done in Scopus Indexing journal, and one funding project proposal is been proposed and sent to send Visvesvaraya Technological University, Public university in Belgaum, Karnataka, One Indian patent is been published. She is a life member of Computer Society of India (CSI), Lattice Science Publication (LSP) and International Association of Engineers (IAENG). 